increased by $62 \%$ \& $46 \%$ respectively from Group A to B although no evidence of any significant change in the proportion of patients having surgery compared with DXT was seen between Group A/B $(\mathrm{p}=0.75)$. Compared with 2005 $(\mathrm{n}=18), 29$ patients underwent surgery in $2012(\mathrm{p}=0.08)$. The proportion of wedge/segmentectomy resections (W/S) doubled over time although this change did not reach significance $(p=0.22)$. Similar non-significant changes in the proportion of surgical patients with stage 1 disease $(\mathrm{p}=0.92)$, post-operative N2 disease $(\mathrm{p}=0.81)$ and pre-operative histology $(\mathrm{p}=0.58)$ were observed between Group A/B. In contrast, the rate of pre-DXT histology increased significantly from $34 \%$ to $65 \%$ ( $\mathrm{p}=0.0007)$. Overall concordancy between pre-operative and final histology was $97 \%$. In 6 patients not having preoperative histology, the final diagnosis was malignant neuroendocrine tumour.

Conclusions Improvements in the SLCS over the last 4 years have led to parallel rises in surgical and DXT rates. The rise in surgery numbers likely reflects better patient selection and increase in use of W/S whereas that for DXT appears to be the result of improved diagnostics reflected by significantly higher rates of histological diagnosis.

Abstract P7 Table 1. Differences between Group A (20052008) vs. Group B (2009-2012)

\begin{tabular}{llllllll}
\hline Year & Surgery & DXT & W/S & T1 Surgery & N2 & $\begin{array}{l}\text { Post-op } \\
\text { histology }\end{array}$ & $\begin{array}{l}\text { DXT } \\
\text { histology }\end{array}$ \\
\hline Group A & 68 & 56 & $4(6 \%)$ & $24(35 \%)$ & $10(15 \%)$ & $20(29 \%)$ & $19(34 \%)$ \\
Group B & 110 & 82 & $14(13 \%)$ & $41(37 \%)$ & $19(17 \%)$ & $38(36 \%)$ & $53(65 \%)$ \\
Total & 178 & 138 & $18(10 \%)$ & $65(37 \%)$ & $29(16 \%)$ & $58(33 \%)$ & $72(52 \%)$ \\
\hline
\end{tabular}

\section{P8 THERMAL ABLATION OF PULMONARY MALIGNANCIES: SURVIVAL, TECHNICAL SUCCESS AND COMPLICATIONS}

MW Little, DYF Chung, P Boardman, FV Gleeson, EM Anderson; Department of Radiology, Churchill Hospital, Oxford, England

\subsection{6/thoraxjnl-2013-204457.158}

Objectives Survival analysis, technical success, safety and imaging follow-up of malignant pulmonary nodules treated with microwave and radio-frequency ablation.

Materials/Methods Between July 2010 and July 2012, 28 patients, 14 female, mean age 61 years (31-87) with 54 pulmonary malignancies of mean diameter $18 \mathrm{~mm}(6-59 \mathrm{~mm})$ underwent computed tomography (CT)-guided thermal ablation (radio-frequency ablation for two lesions, microwave ablation for the remainder). Bronchogenic carcinoma was treated in 15 patients, metastatic tumour in the remainder (tumours were diagnosed by biopsy (67\%) and or PET/CT). Technical success was defined as needle placement in the intended lesion without death or serious injury. Adequacy of ablation was assessed at 24 hours on contrast-enhanced CT. Circumferential solid or ground glass opacification $>4 \mathrm{~mm}$ was deemed adequate, and $>5 \mathrm{~mm}$ was deemed ideal. Patients were followed with contrastenhanced CT 3 monthly until death, or local tumour recurrence; Recurrence was identified by enlargement of the zone, the development of contrast enhancement in part of the zone, or a change in the shape of the ablation zone as a result of enlargement of one area. Survival rate was evaluated by Kaplan-Meier analysis
Results Thermal ablation was technically successful in $98 \%$ $(\mathrm{n}=50)$. Mean ablation duration was 5.2 minutes (1-24 minutes). 19(68\%) patients developed a pneumothorax post procedure, $7(25 \%)$ required a chest drain. 30-day mortality rate was $0 \%$. The mean hospital stay was 1.3 days (1-7 days). Local recurrence was only identified in one patient at a median follow up of 12 months. The 1-year survival for all cause mortality was $68 \%$; cancer-specific mortality yielded a 1 -year survival of $75 \%$.

Conclusions Thermal ablation of pulmonary malignancies is a safe, successful technique. Local control rates and survival analysis are encouraging, with rapid treatment times, performed as a single day-case procedure advantageous over stereotactic beam radiotherapy.

\section{P9 EPIDEMIOLOGICAL SURVEY SUGGESTS LUNG CANCER IS LESS COMMON IN PATIENTS WITH HEREDITARY HAEMORRHAGIC TELANGIECTASIA COMPARED TO CONTROLS}

${ }^{1} \mathrm{AE}$ Hosman, ${ }^{2} \mathrm{HL}$ Devlin, ${ }^{3} \mathrm{BM}$ Silva, ${ }^{3} \mathrm{AH}$ Elphick, ${ }^{3} \mathrm{CL}$ Shovlin; ${ }^{1}$ Academic Centre of Amsterdam and Imperial College London, Amsterdam and London, NL and UK; ${ }^{2} B$ arts and the London School of Medicine, and Dentistry and Imperial College London, London, UK; ${ }^{3}$ Imperial College London, London, UK

\subsection{6/thoraxjnl-2013-204457.159}

Background Over 41,000 people are diagnosed with lung cancer annually in the UK with only $8 \%$ of men, and $9 \%$ of women surviving at least 5 years after diagnosis. Antibodies against the endothelial cell protein endoglin are proving promising in early clinical trials [1]. Many individuals with hereditary haemorrhagic telangiectasia (HHT) have inherited endoglin mutations. This autosomal dominant trait affects approximately 1 in 5,000 people, causing multi-systemic vascular lesions. We hypothesised that lung cancer rates may differ in individuals with HHT.

Methods To provide sufficient power to compare lung cancer rates in HHT patients and controls, we developed a questionnaire capturing data on multiple relatives per respondent, powered to detect differences in lung cancer rates. Blinded to cancer responses, reports of HHT-specific features allowed assignment of participants and relatives as HHT-subject, unknown, or control. Logistic and quadratic regression were used to calculate crude and age-adjusted odds ratios.

Results By data download on 30.6.2012, 1,307 participants (including 1,012 HHT-subjects, 142 controls) completed the questionnaire. Ages (medians 55/53ys), gender (65/65\% female) and general demographics were similar. The number of current/ former smokers did not differ significantly between HHT and control groups $(\mathrm{p}=0.38)$, but the HHT arm reported significantly greater pack year smoking histories than the controls (Mann Whitney $\mathrm{p}=0.01)$. Combining data of participants and relatives resulted in a control-arm of 2,817 (52\% female), and HHT-arm of 2,166 (58\% female). Median ages were77ys [IQR 65-82] and 66ys [IQR 53-77] respectively. Rates of 15 cancers predominantly recognised as primary cancers in the control group generally matched the age standardised frequency of the general population. The ratio of observed/expected incidence was significantly higher for cancers at common sites of metastatic spread (lung, liver and brain). As expected cancer rates increased with age ( $\mathrm{p}<0.0001$, all cancers). In both crude and age-adjusted regression, lung cancers were significantly less frequent in the HHT arm compared to controls (age-adjusted odds ratio $0.48[0.30,0.70], \mathrm{p}=0.0012)$. 
Conclusions Individuals with HHT may be protected against primary and/or metastatic lung cancer. This does not appear to be due to reduced smoking habit.

\section{REFERENCES}

1. Bernabeu and Shovlin, Thomson Reuters Pharma (21 December 2011) [Online].

\section{P10 CARBOPLATIN AND GEMCITABINE IMPAIR NEUTROPHIL PHAGOCYTIC FUNCTION}

CR Popplewell, M-H Ruchaud-Sparagano, J Scott, PA Corris, AJ Simpson; Newcastle University, Newcastle-upon-Tyne, UK

\subsection{6/thoraxjnl-2013-204457.160}

Background Lung cancer causes 6\% of UK deaths with 20\% due to infection ${ }^{1,2}$. The reason is multifactorial with myelosuppression secondary to chemotherapy being a contributor. However, the effect of chemotherapy drugs on the function of peripheral neutrophils has rarely been explored. It is proposed carboplatin and gemcitabine may decrease the function of circulating neutrophils that are present, causing a poorer response to infection by neutrophils that are present. Granulocyte-macrophage colony-stimulating factor (GM-CSF) is used to stimulate neutrophil production in febrile neutropenic patients on chemotherapy, however studies show it also improves mature neutrophil function ${ }^{3,4}$. The aim was to research the effect of carboplatin and gemcitabine on neutrophil phagocytic function and to determine whether GM-CSF reverses any deficit seen in phagocytosis.

Methods Neutrophils isolated from healthy donor blood by dextran sedimentation followed by discontinuous Percoll gradient. Untreated neutrophils compared with those pre-incubated with carboplatin, gemcitabine or both drugs $\left(10^{-4} \mathrm{mM}\right)$. Phagocytosis assessed by ingestion of serum-opsonised zymosan. Repeated with 30 minute GM-CSF (5 ng/ml) pre-incubation. Apoptosis analysed by flow cytometry. All cells incubated for 2 hours untreated or with single or both chemotherapeutic agents $\left(10^{-4}\right.$ $\mathrm{mM}$ ) or phorbol 12-myristate 13-acetate (PMA) for 30 minutes. Cells stained with APC-Annexin V to detect apoptosis and propidium iodide to distinguish early and late apoptotic cells.

Results A significant decrease in percentage phagocytosis occurred with chemotherapy drugs compared to untreated neutrophils: carboplatin $\mathrm{p}=<0.001$, gemcitabine $\mathrm{p}=<0.01$, carboplatin and gemcitabine $\mathrm{p}=<0.05$. GM-CSF caused a significant increase in percentage phagocytosis when gemcitabine caused a deficit $(\mathrm{p}=<0.05)$ with a 9-13\% increasing trend seen in other conditions. No difference in apoptosis occurred between untreated (median 3.9\%) and chemotherapy treated neutrophils (median: carboplatin 4.4\%, gemcitabine 4.9\%, carboplatin and gemcitabine 3.6\%).

Conclusions This study demonstrated that phagocytosis is impaired by carboplatin and gemcitabine in healthy volunteer blood. Therefore it is proposed a reduced response to infection may also occur in lung cancer patients given these drugs, contributing to mortality. There was no difference in apoptosis, suggesting an alternative mechanism of action. Further study is required to explore the mechanism of action and the effect of GM-CSF on phagocytosis following chemotherapy.

\section{REFERENCES}

1. Cancer Research UK. 2012 http://www.cancerresearchuk.org/cancer-info/cancerstats/ types/lung/mortality/

2. Nichols L et al. Arch Path Lab Med 2012;136:1552-7

3. American Society of Clinical Oncology. JCO 2006;24:3187-205

4. Bober LA et al. Immunopharmacology 1995;29:111-9
P11 TYROSINE KINASE INHIBITOR USE IN PULMONARY ADENOCARCINOMA

G Jones, M Murthy, D Komrower, N Hunt, M Ledson, M Walshaw; Liverpool Heart \& Chest Hospital, Liverpool, United Kingdom

10.1136/thoraxjnl-2013-204457.161

Introduction Personalised treatments are becoming increasingly important in medicine, and the use of tyrosine kinase inhibitors (TKI) in the management of the subset of patients with pulmonary adenocarcinomas which express a mutation in the epidermal growth factor receptor (EGFR), is one example of this approach. However, although this cell type is common, in the UK only a small proportion of patients express the EGFR mutation and there is little published data on the number of patients receiving TKI therapy. Since 2010 we have routinely performed EGFR testing on pulmonary adenocarcinoma samples at our large cancer unit and were interested to assess our use of TKIs.

Methods We reviewed all cases of pulmonary adenocarcinoma since EGFR testing began, looking for the proportion EGFR positive, their performance state (PS), and what treatments were offered.

Results Of 241 cases of primary pulmonary adenocarcinoma, 54 $(22 \%)$ had insufficient material available for mutation testing. Of the remaining 187, $23(12 \%)$ were EGFR positive (mean age 76 [range 47-92], 18 female). Although 16 were PS $\leq 2$, only 5 patients had stage $1 \mathrm{~A}$ cancer with 13 having advanced disease (stage 3B/4). Seven patients underwent attempted curative surgery and 4 patients with poor PS were managed with best supportive care only. Twelve patients $(52 \%$ of the EGFR positive group and $6.4 \%$ of the tested cohort) received a TKI (mean age 74 [50-92], PS $\leq 2$ in 8,10 female), and in 3 of these it was the only treatment modality offered (1\%).

Conclusions We have shown that our cohort of patients with adenocarcinoma had a higher rate of EGFR mutation than expected, and that half of these received targeted biological therapy with tyrosine kinase inhibitors. Importantly TKI therapy was the only treatment modality available for patients who would have previously been untreated. This work emphasises the need to obtain a histological diagnosis in patients with lung cancer, to ensure that all possible treatment modalities can be considered.

\section{P12 TEMPORAL TRENDS, CAUSES \& RISK FACTORS FOR HOSPITAL ADMISSIONS IN INCURABLE LUNG CANCER}

${ }^{1} \mathrm{M}$ Shah, ${ }^{2} \mathrm{SCO}$ Taggart; ${ }^{1}$ University of Manchester, Manchester, UK; ${ }^{2}$ Salford Royal NHS Foundation Trust, Salford, UK

10.1136/thoraxjnl-2013-204457.162

Introduction Little is known about hospital admissions (HAs) following a diagnosis of incurable lung cancer (LC). This study sets out to identify temporal trends for HA in this group of vulnerable patients in addition to exploring the reasons behind and potential risk factors for HA.

Methods All new LC diagnoses for 2009-2011 ( $\mathrm{n}=565)$ were identified, from which 1:4 were selected randomly $(n=142)$. Records were reviewed and those patients treated with curative intent or diagnosed and died in same HA were excluded from analysis. Basic demographic data were collected including comorbidity score, stage, histology and LC was classified as either central (mediastinum to origin of lobar bronchi and vessels) or 